Nippos Saisen Gakktiekin a4(1), 82-87 (1998)

\title{
低温殺菌条件下で調製したオボムコイド添加 魚肉的品の物性特性
}

\author{
比山雅昭, 三代拄也, 掫正泰之, 中川巷子

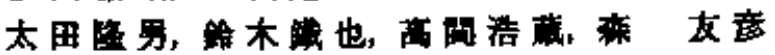

(1996年11月28 Bsity)

Phytical Propertics of Kamaboko with Owomucoid

Fasteturized at Several Temperatares

Masagki Sugiyama,"1 Tatsxya Mishiro,"1 Yasquyuli Tsukamasa, ,1

Kyolo Nalagawa, *2 Takao Ota, *2 Tetsuya Suzuki, *3

Kozo Takama, and Tomnhiko Mori"*t

\begin{abstract}
Fish gels were hented for varions times at various temperaturea from 60 to $75 \mathrm{C}$, whose aterilip ing effect were tbe samp as the stondard $(75 \mathrm{C})$ set by the Japan Food Samitation Isw. Textural

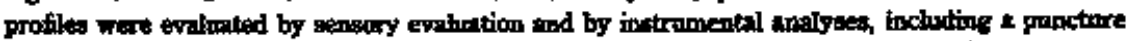
test, an ordinary texture test and a bect by a texhre mapping enolyzer system (TMAS). The reonlts of the puncture terto weins apherical and weitge-shaped plimgera were different from each other. In the texcure test, the somples differed only in hardiness and the differesces were stintilar to those obtatined in the puncenre teat ucing a spherical phunzer. According to the TMAS analyde, the patterns

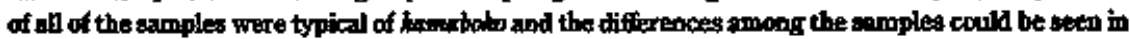
the parameters of CW.F as bardnees, RS as elesticity and CM as ghootness. Regerding the rebation between sensory evaluation and instrumental evaluation, the TMAS realls were most highty correlated with the enensory epatistion.
\end{abstract}

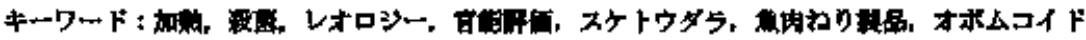

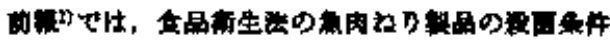

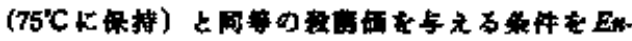

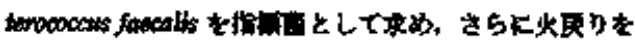

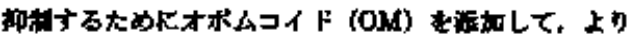

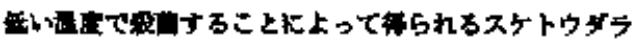

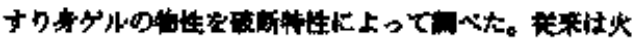

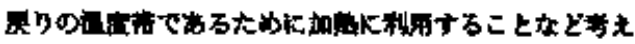

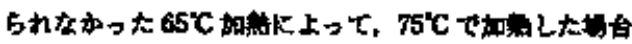

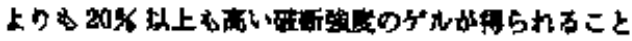

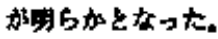

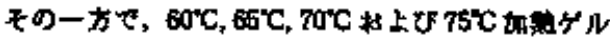

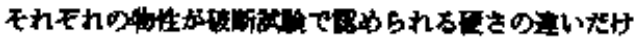

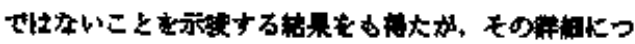
いで明らがでなかった。

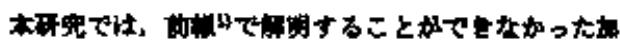

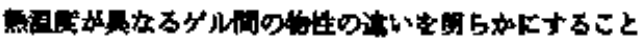

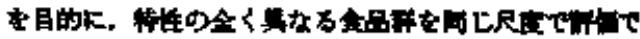

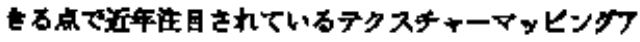

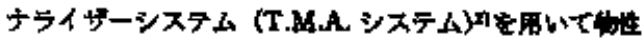

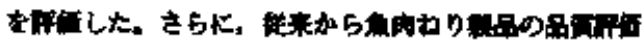
に用いられている

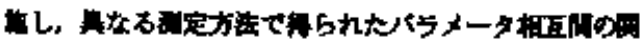
和よついてい国ら加にようとした。

\section{究的法}

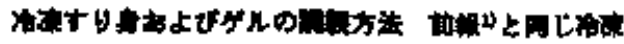

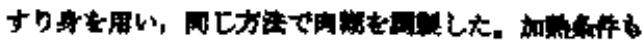

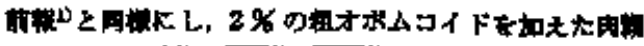

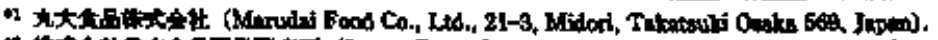

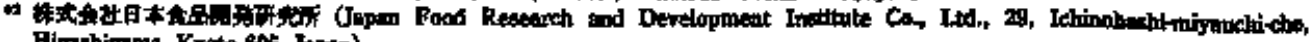
Hiposhipams, Koto B0i, Kapas).

4 A:

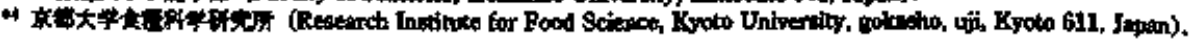




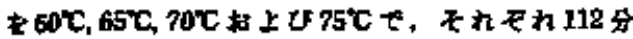

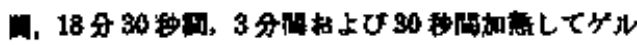
LLた.

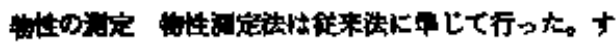

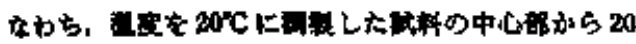

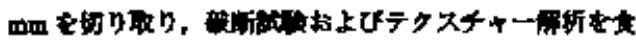

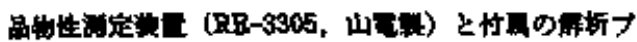

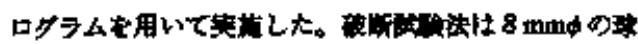

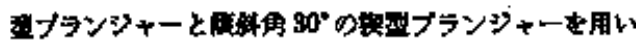

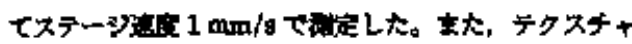

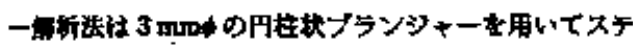

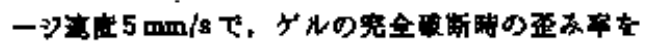

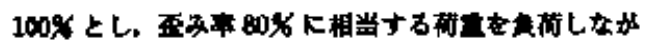

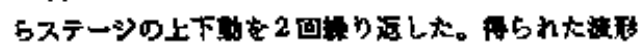

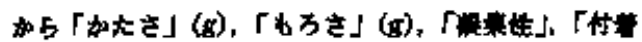

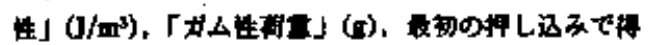

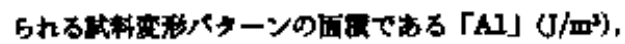

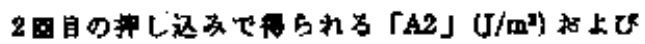

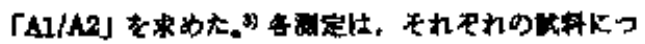

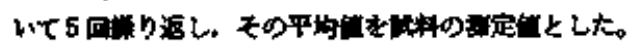

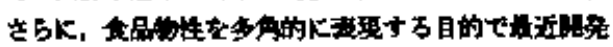

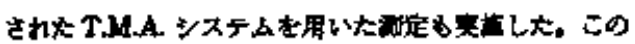

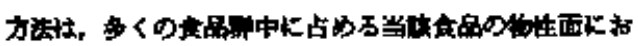

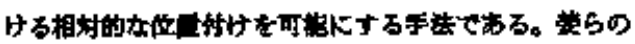

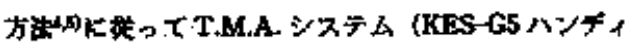

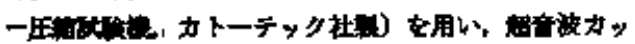

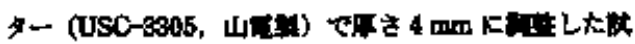

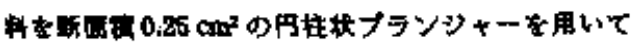
$4.8 \mathrm{~mm} / \mathrm{eec}$ O

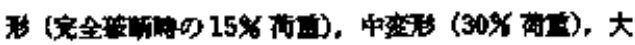

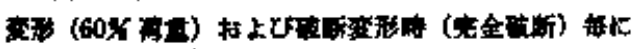

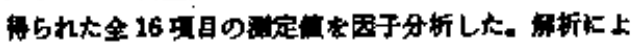

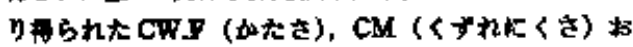

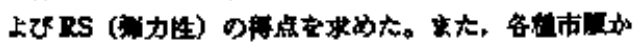

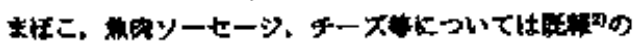

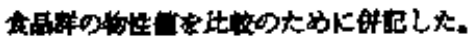

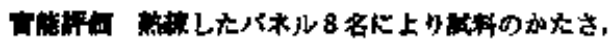

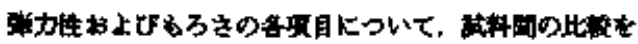

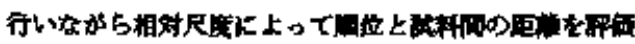

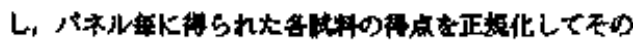

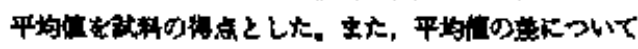

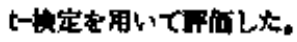

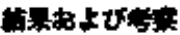

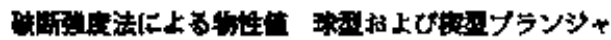

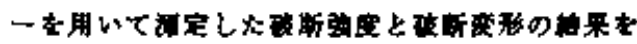

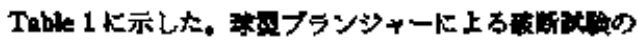

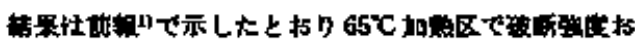

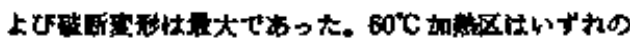

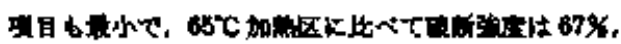

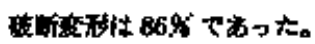

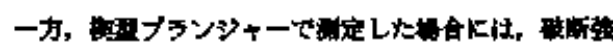

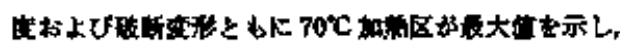

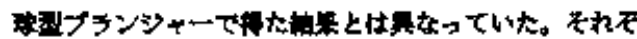

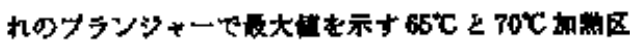

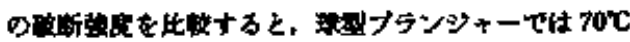

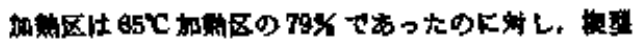

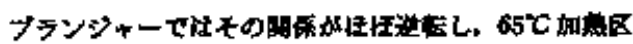

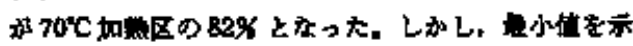

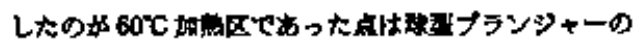

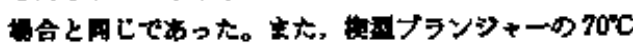

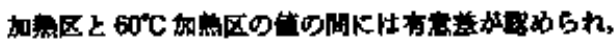

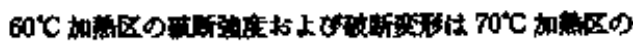
スれのモんモれ70\%お上け87\%ですった。

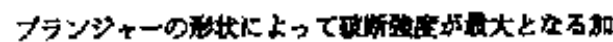

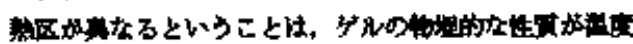

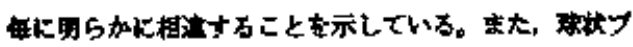

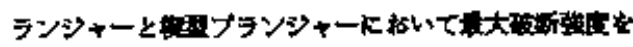

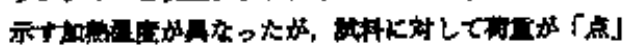

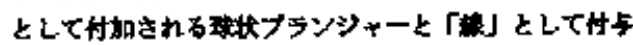

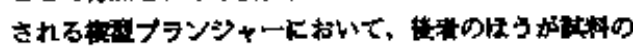

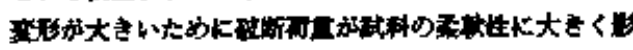

Twble 1. Functure test by using different planger for fish gels with owomactoid

\begin{tabular}{|c|c|c|c|c|c|c|c|c|}
\hline \multirow{3}{*}{ Heat conditione } & \multicolumn{4}{|c|}{8 mmo sphericel type } & \multicolumn{4}{|c|}{$30^{4}$ wedore type } \\
\hline & \multicolumn{2}{|c|}{$(g)$} & \multicolumn{2}{|c|}{ (mm) } & \multicolumn{2}{|c|}{ (s) } & \multicolumn{2}{|c|}{$(\mathrm{mm})$} \\
\hline & menn & ad. & mean & g.d. & mean & ad. & mean & a.d. \\
\hline $600^{\circ}-112$ min & \multicolumn{2}{|c|}{$675.0 \pm 39.0$} & \multicolumn{2}{|c|}{$15.2 \pm 0.4$} & \multicolumn{2}{|c|}{$898.7 \pm 129.0^{4}$} & \multicolumn{2}{|c|}{$11.6 \pm 0.6$} \\
\hline $65 \mathrm{C}-18.5 \mathrm{~min}$ & \multicolumn{2}{|c|}{$1008.0 \pm 114.2$} & \multicolumn{2}{|c|}{$17.6 \pm 0.7$} & \multicolumn{2}{|c|}{$1045.3 \pm 102.6^{b}$} & \multicolumn{2}{|c|}{$12.5 \pm 0.14$} \\
\hline 7orc- 3 min & \multicolumn{2}{|c|}{$794.7 \pm 20.50$} & \multicolumn{2}{|c|}{$16.7 \pm 0 . A^{b}$} & \multicolumn{2}{|c|}{$1279.0 \pm 101.2^{6}$} & \multicolumn{2}{|c|}{$13.3 \pm 0.2^{\mathrm{bc}}$} \\
\hline $750 \mathrm{C}-6.5 \mathrm{~min}$ & \multicolumn{2}{|c|}{$819.3 \pm 79.5^{\circ}$} & \multicolumn{2}{|c|}{$16.4 \pm 0.4^{\circ}$} & \multicolumn{2}{|c|}{$1182.7 \pm 82.9^{\circ}$} & \multicolumn{2}{|c|}{$12.9 \pm 0.2^{*}$} \\
\hline
\end{tabular}

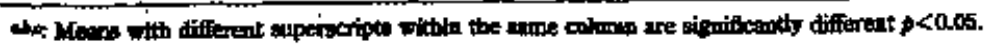
$(0=5)$ 
Thble 2. Fhyacal properties-of flah geds with orompeold obtalaed woder varlowis heat condifions

\begin{tabular}{|c|c|c|c|c|}
\hline \multirow{2}{*}{ Beat conditions } & $\begin{array}{c}\text { Hardiness } \\
(g)\end{array}$ & $\begin{array}{c}\text { Britheness } \\
\text { (g) }\end{array}$ & Cohesiresess & $\begin{array}{c}\text { Adhogivens } \\
\left(\mathrm{J} / \mathrm{m}^{\mathrm{s}}\right)\end{array}$ \\
\hline & mens $0 . d$. & mean s.d. & meng & mean \\
\hline $60 \mathrm{C}-112 \mathrm{~min}$ & $176.0 \pm 8,0^{\circ}$ & $54.3 \pm 5.34$ & $0.63 \pm 0.03$ & $7.1 \times 10^{4} \pm 9.7 \times 10^{3}$ \\
\hline $65 \mathrm{C}-18.5 \mathrm{~min}$ & $217.3 \pm 6.8^{6}$ & 50.0土 g.t. & $0.51 \pm 0.0 \sigma^{2}$ & $6.1 \times 10^{4} \pm 2.5 \times 10^{3}$ \\
\hline $70^{\circ} \mathrm{C}-3 \mathrm{~min}$ & $185.0 \pm 6.7=$ & $57,0 \pm 13.9$ & $0.51 \pm 0.02$ & $5.8 \times 10^{4} \pm 3.3 \times 10^{n}$ \\
\hline \multirow[t]{3}{*}{$75 \div \mathrm{C}-0.5 \mathrm{~min}$} & $197.0 \pm 8.3^{*}$ & $75.3 \pm 15.8$ & $0.48 \pm 0.06^{n}$ & $5.4 \times 10^{4} \pm 7.6 \times 10^{2}$ \\
\hline & $\frac{G(g)}{(g)}$ & $\left(\mathrm{Al} / \mathrm{m}^{\mathrm{J}}\right)$ & $\underset{\left(\mathbf{J} / \mathbf{m}^{\mathrm{b}}\right)}{\mathbf{A}}$ & $A 1 / A 2$ \\
\hline & mean $8 . \mathbf{d}_{+}$ & mean & mean & meath \\
\hline $60{ }^{\circ} \mathrm{C}-112 \mathrm{~min}$ & $91.0 \pm 4.1$ & $3.6 \times 10^{5} \pm 2.1 \times 10^{42}$ & $1.9 \times 10^{6} \pm 1.0 \times 10^{62}$ & $0.517 \pm 0.029$ \\
\hline $65 \mathrm{C}-18.5 \mathrm{~min}$ & $99.3 \pm 21.5$ & $40 \times 10^{5} \pm 2.4 \times 10^{4 x}$ & $2.0 \times 10^{4} \pm 2.4 \times 10^{4}$ & $0.513 \pm 0.054$ \\
\hline $700^{\circ} \mathrm{C}-3$ min & $94.6 \pm 4.6^{\circ}$ & $3.6 \times 10^{5} \pm 9.4 \times 10^{2}=$ & $1.8 \times 10^{4} \pm 12 \times 10^{40}$ & $0.504 \pm 0.026$ \\
\hline $7^{\circ} \mathrm{C}-0.5 \mathrm{~min}$ & $95.5 \pm 9.5$ & $8.8 \times 10^{5} \pm 1.8 \times 10^{4}=$ & $1.9 \times 10^{5} \pm 1.9 \times 10^{4}$ & $0.485 \pm 0.04 \%$ \\
\hline
\end{tabular}

さされためと节られた。

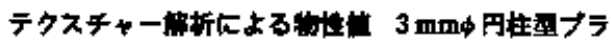

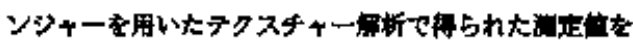

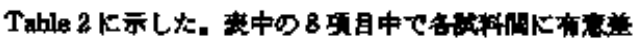

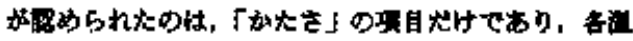

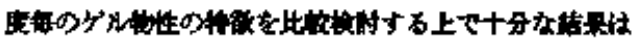

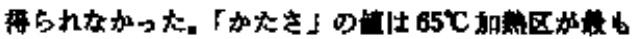

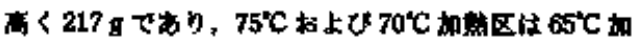

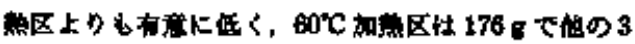

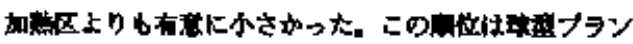

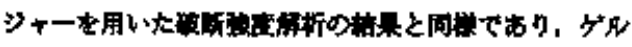

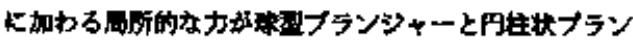

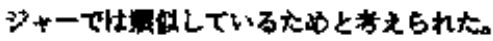

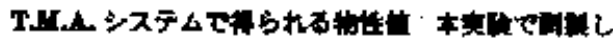

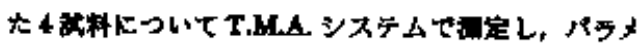

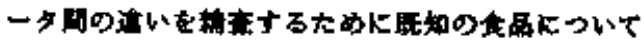

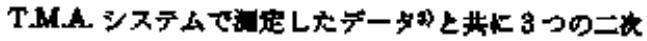

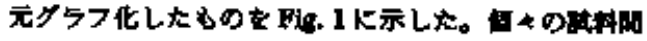

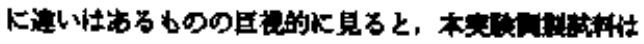

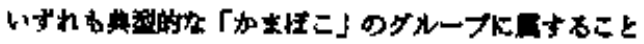

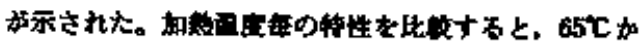

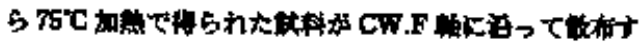

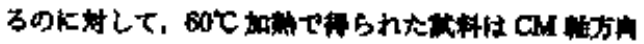

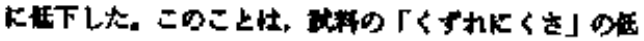

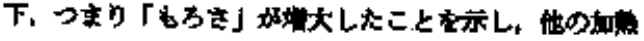

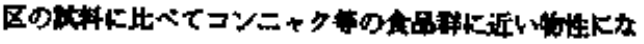

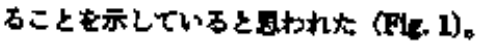

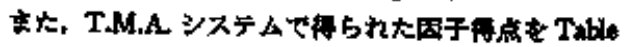
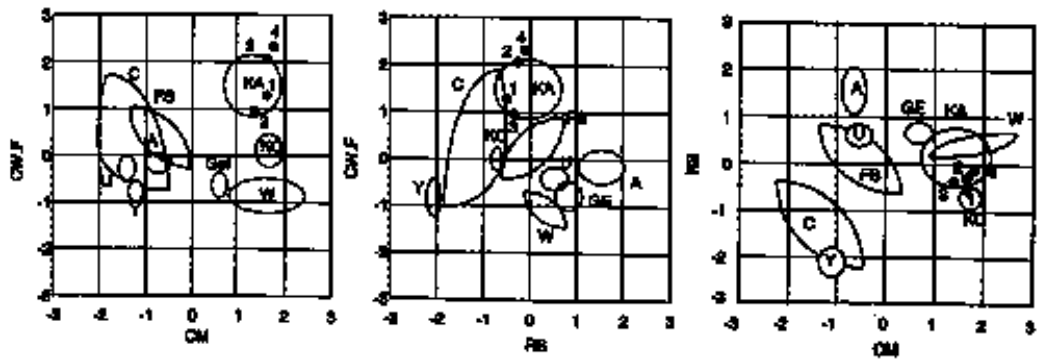

Fis. 1. Texture map of the fiah gels ind varions other toods.

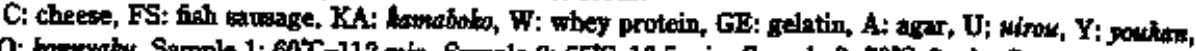

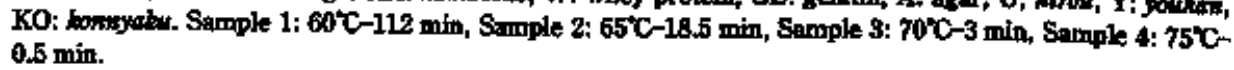


Tahle 5. T.M. factar score and serworg score of fish geks with ovomucria

\begin{tabular}{|c|c|c|c|c|c|c|}
\hline \multirow{3}{*}{ Beat concitions } & \multicolumn{3}{|c|}{ T.M. factor goore } & \multicolumn{3}{|c|}{ Sensory acore } \\
\hline & \multirow{2}{*}{ CW. F } & \multirow{2}{*}{$\mathrm{CM}$} & \multirow{2}{*}{ RS } & Haruness & Eloeticity & Shortneas \\
\hline & & & & mean s.d. & mean & mean \\
\hline $600^{\circ}-112 \mathrm{~min}$ & 1.298 & 1.686 & -0.492 & $-1.19 \pm 0.32^{2}$ & $-0.70 \pm 0,35$ & $0.96 \pm 0.86$ \\
\hline $65{ }^{\circ} \mathrm{C}-18.5 \mathrm{~min}$ & 2.075 & 1.697 & -0.262 & $4.13 \pm 0.55^{b}$ & $0,23 \pm 0,9 \mathrm{~g}^{\mathrm{b}}$ & $-0.50 \pm 0.596$ \\
\hline $70 \mathrm{C}-3$ min & 0.971 & 1.968 & -0.344 & $0.01 \pm 0.98^{*}$ & $-0.11 \pm 1.07^{\star}$ & $0.29 \pm 0.69$ \\
\hline $75 \mathrm{C}-0.5 \mathrm{~min}$ & 2.384 & 1.777 & -0.050 & 1. $15 \pm 0.32$ & $0.59 \pm 0.65$ & $-0.75 \pm 0.70^{\circ}$ \\
\hline
\end{tabular}

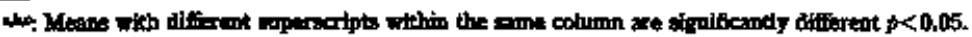

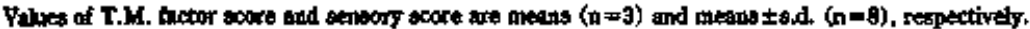

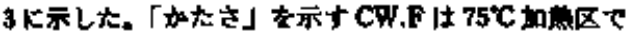

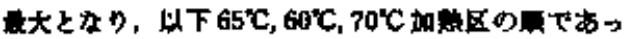

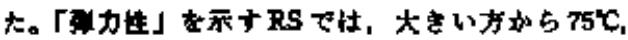

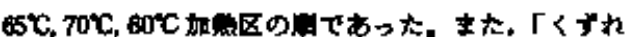
Kくさ」を英すCM 恃大きい方加ら $75^{\circ} \mathrm{C}, 65^{\circ} \mathrm{C}, 60^{\circ} \mathrm{C}$,

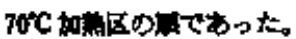

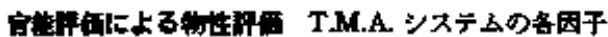

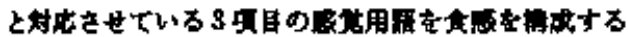

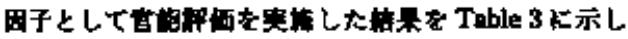

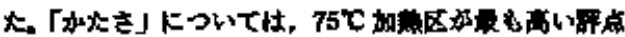

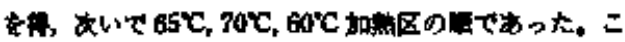

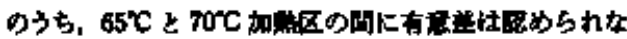

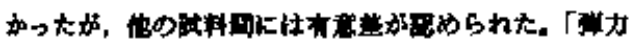

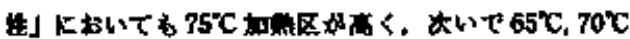

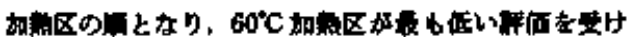

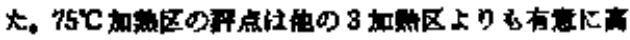

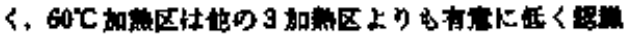

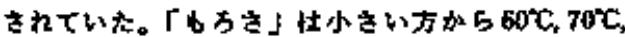

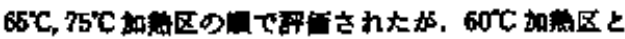

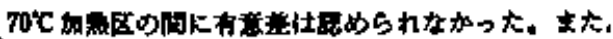

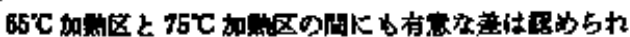

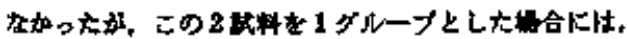

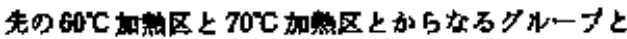

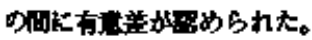

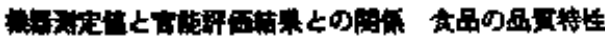

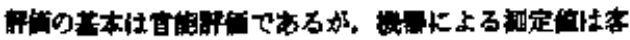

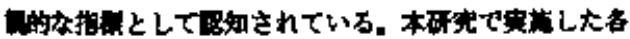

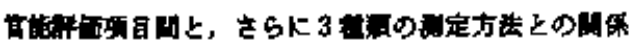

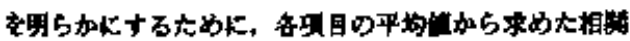

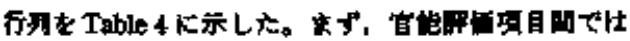

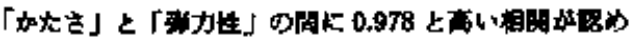
られた。一方、「するき」は奻たさ」との回に一0.927。

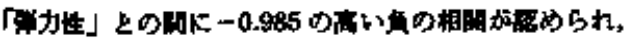

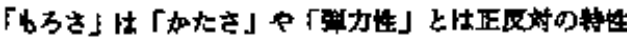

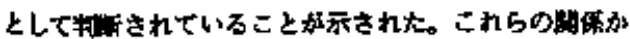

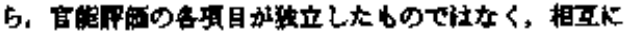

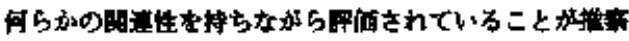
さnた。

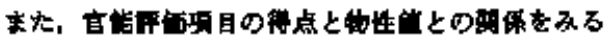

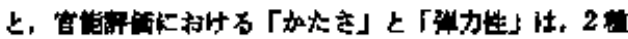

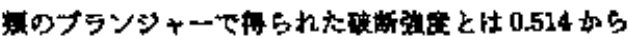

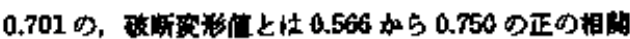

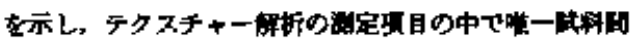

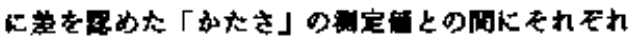
0.557 拉上び0.707 の正の相国西示した。

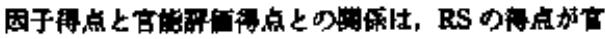

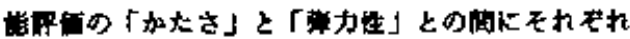

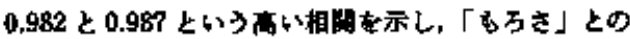

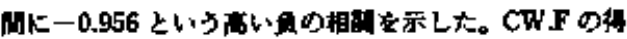

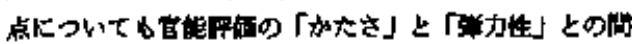

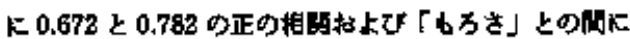
-0.842 といっ出の相则を示した。一力，CM D得点と

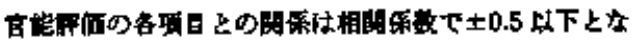

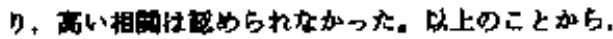

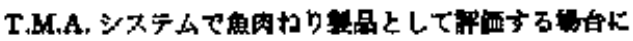

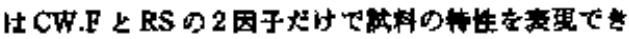

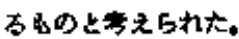

T.MA. ジステムを用いて可定した市邽かま标こにお

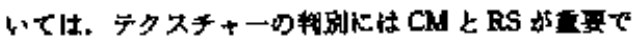

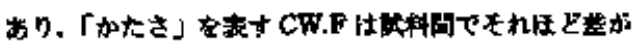

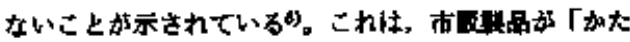

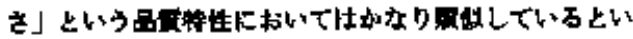

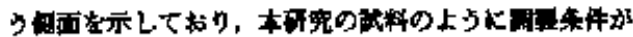

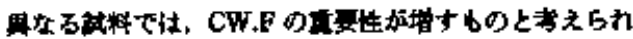

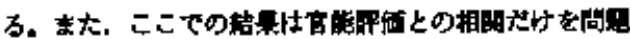

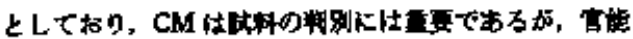

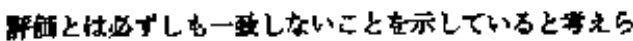
机布。

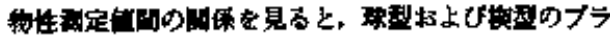

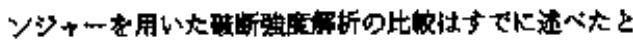


Tuble 4. Coxrelation coefficient matrix between scores or vahued of sensony evalustion and physical menourements

\begin{tabular}{|c|c|c|c|c|c|c|c|c|c|c|c|c|c|c|c|c|c|c|}
\hline & & \multicolumn{3}{|c|}{8 8ng evaluation } & \multicolumn{2}{|c|}{ 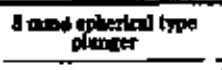 } & \multicolumn{2}{|c|}{ 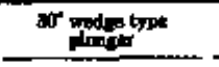 } & \multicolumn{8}{|c|}{ Textriat } & \multicolumn{2}{|c|}{ than } \\
\hline & & Hantur & Thenty s & Shoormess & Pantion I & Detergative & pored (b) & Dention & Honden & 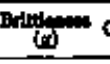 & 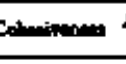 & 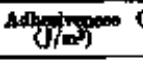 & 6 & $\sin 1$ & $\left(f^{2}\right)$ & Al/as & $\overline{C W . P}$ & cru \\
\hline 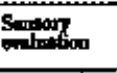 & $\begin{array}{l}\text { ghentivy } \\
\text { Shoteon }\end{array}$ & $\begin{array}{r}0.675 \\
-0.82 z\end{array}$ & $-\overline{0.085}$ & $\overline{-}$ & $\bar{z}$ & $\bar{z}$ & $\overline{-}$ & $\bar{z}$ & - & - & $\overline{-}$ & I & $=$ & $\begin{array}{l}- \\
- \\
\end{array}$ & \pm & $\overline{-}$ & $\bar{z}$ & $\overline{-}$ \\
\hline \multirow{2}{*}{ Anger } & Prencture forct & 0.514 &. .660 & -0.7 붕 & - & - & - & - & - & - & - & - & - & - & - & - & - & - \\
\hline & 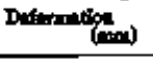 & 0.566 & 0.05 & -0.711 & D.964 & - & - & - & - & - & - & - & - & - & - & - & - & - \\
\hline \multirow{2}{*}{ SW } & Punctuet fon & 0.701 & $0.5 \%$ & -0.454 & 0.200 & 0.457 & - & - & - & - & - & - & - & - & - & - & - & - \\
\hline & Detomention (ton) & 0.75 & .652 & -0.555 & 0.370 & 0.69 & D. & - & - & - & - & - & - & - & - & - & - & $=$ \\
\hline \multirow[t]{5}{*}{ Tats } & 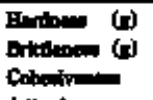 & $\begin{array}{r}0.467 \\
0.701 \\
-0.864\end{array}$ & $\begin{array}{r}0.707 \\
0.000 \\
-0.077\end{array}$ & $\begin{array}{l}-0.811 \\
-0.010 \\
0.650\end{array}$ & $\begin{array}{l}0.072 \\
-0.912 \\
-0.518\end{array}$ & $\begin{array}{r}0.013 \\
-0.198 \\
-0.547\end{array}$ & $\begin{array}{r}0.111 \\
0.67 \\
-0.609\end{array}$ & $\begin{array}{c}0.972 \\
0.372 \\
-0.610\end{array}$ & $-\overline{-a .0}$ & $\overline{-}$ & $\bar{I}$ & $\begin{array}{l}- \\
-\end{array}$ & $\underline{-}$ & $\begin{array}{l}- \\
-\end{array}$ & $\bar{z}$ & $\begin{array}{l}- \\
- \\
-\end{array}$ & $\bar{z}$ & $=$ \\
\hline & 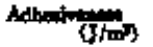 & -0.074 & -0.920 & D.S45 & -0.006 & -0.086 & -0.044 & -0.89 & -0.468 & -0.652 & 0.991 & - & - & - & - & - & - & - \\
\hline & 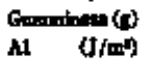 & 0.608 & $\begin{array}{l}0.730 \\
0.747\end{array}$ & $\begin{array}{l}-0.654 \\
-0.0851\end{array}$ & a.901 & 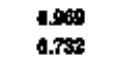 & $\begin{array}{r}0.536 \\
-0,006\end{array}$ & $\begin{array}{l}0.479 \\
0.141\end{array}$ & 1.909 & $\begin{array}{r}-0.098 \\
0.074\end{array}$ & $\begin{array}{l}-0.431 \\
-0.491\end{array}$ & $\begin{array}{l}-0.584 \\
-0.454\end{array}$ & asts & $\bar{z}$ & $\overline{-}$ & $\overline{-}$ & $\overline{-}$ & $\overline{-}$ \\
\hline & $A_{1 / 1 / 3}$ (S/ & 0.68 & 0.251 & -0.16 & ons & $0.0 \pi \mathrm{r}$ & -0.373 & -0.212 & 1.868 & -0.807 & 0.086 & 0.00 & 0.74 & 0.816 & - & - & - & - \\
\hline & $A 1 / A B$ & $-0.8 \%$ & -0.725 & $\mathrm{aGm}$ & -0.0 .5 & -6.111 & -0.8066 & $-0.62 n$ & -0.118 & -0.06 & 0.888 & 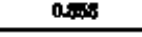 & -0.170 & -0.227 & 0.378 & - & - & - \\
\hline \multirow{2}{*}{ 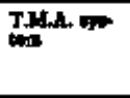 } & $\begin{array}{l}\mathrm{Cr} . \mathrm{P} \\
\mathrm{CA}\end{array}$ & $\begin{array}{l}0.073 \\
0.812\end{array}$ & $\begin{array}{l}0.788 \\
0.205\end{array}$ & $\begin{array}{l}-0.080 \\
-0.080\end{array}$ & $\begin{array}{l}0.556 \\
0.105\end{array}$ & $\begin{array}{r}2.37 \\
-0.14\end{array}$ & $\begin{array}{l}-0.008 \\
-0.006\end{array}$ & $\begin{array}{l}0.057 \\
-0.2006\end{array}$ & $\begin{array}{l}0.711 \\
0.310\end{array}$ & 0 & $\begin{array}{l}-8.094 \\
-8.415\end{array}$ & $\begin{array}{l}-0.450 \\
-0.003\end{array}$ & $\begin{array}{l}0.580 \\
0.102\end{array}$ & 0.0 .668 & $\begin{array}{l}0.514 \\
0.224\end{array}$ & 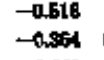 & $\overrightarrow{0.0050}$ & $\bar{z}$ \\
\hline & HB & 090 & 0.987 & - apse & 0.525 & 的1 & 0.666 & 0.612 & $0.60 \mathrm{OH}$ & 0.719 & $-0.07 n$ & -4.923 & a 681 & 0.000 & 0.08 & -0.302 & $0 . m$ & \\
\hline
\end{tabular}




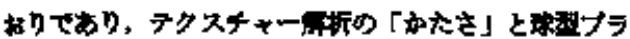

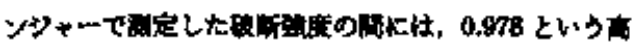

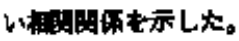

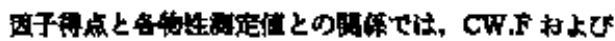

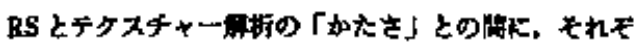

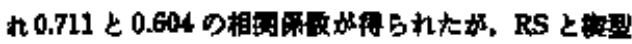

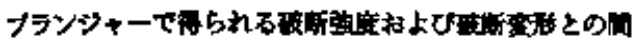

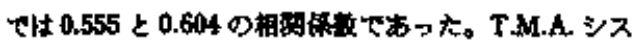

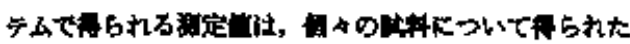

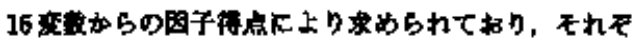

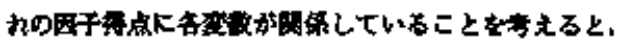

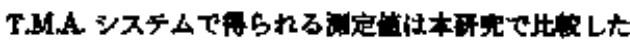

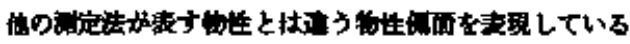
四部生格孝えられた。

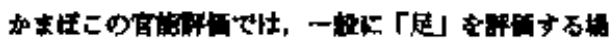

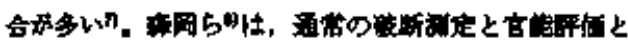

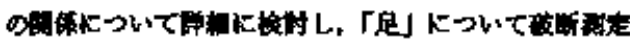

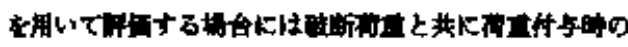

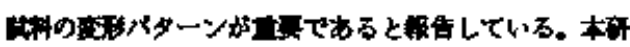

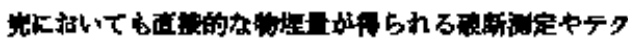
スチャーの析上りる，因子牙析の界析に上

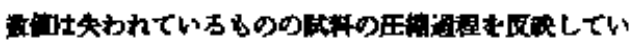

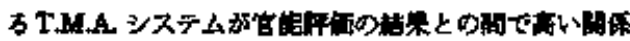

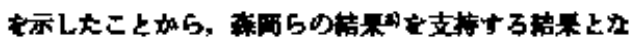

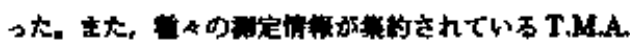

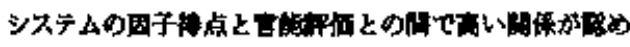

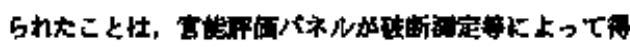

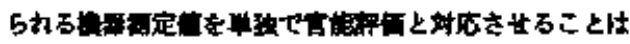

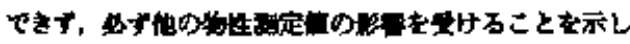

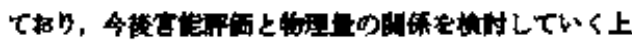

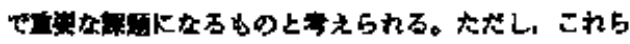

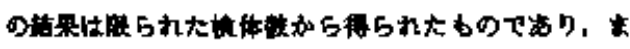

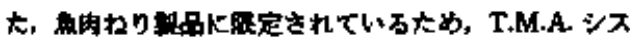

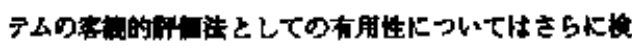

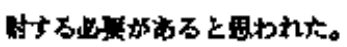

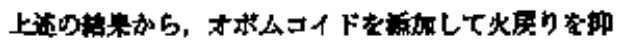

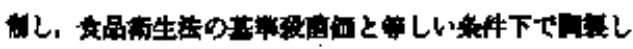

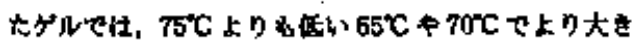

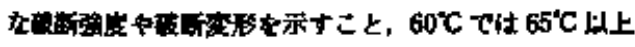

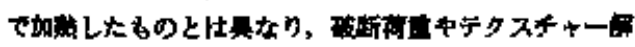

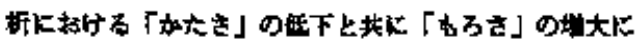

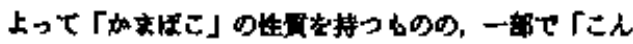

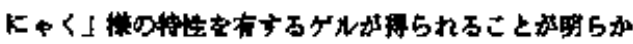

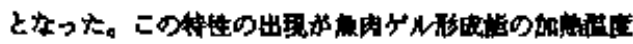

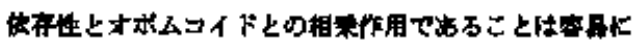

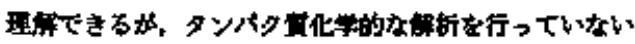

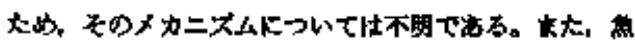

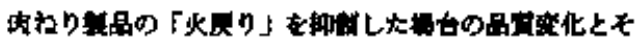

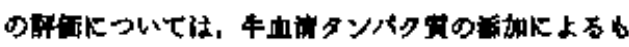

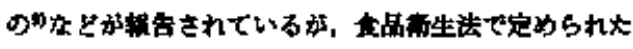

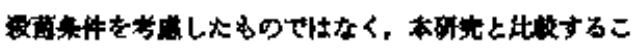
と杜然かい.

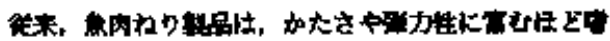

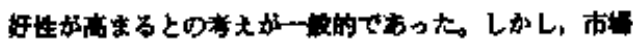

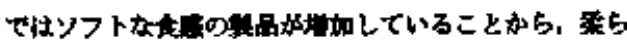

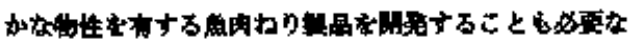

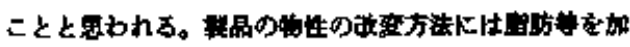

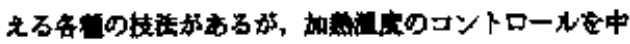

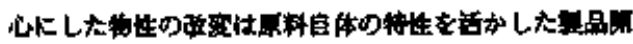

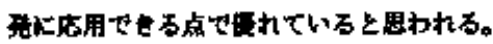

文

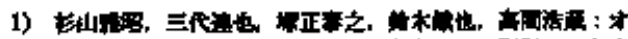

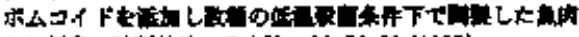

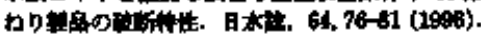

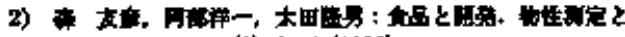
Fクス5+ー, $\mathrm{m}(2), 8-11$ (1996).

3) II. I Kang, Y. Mateumman, and T. Moxi Cheracterization of Textime and Mechromical Propertica of Hextinctured Soy Protein Geln, JAOCS, 48, 339-345 (1993).

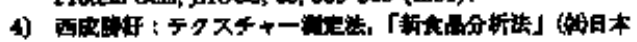

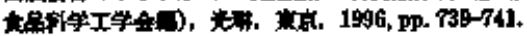

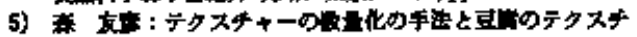
$\rightarrow$ - テイリーフート. 12, 14-19 (1992).

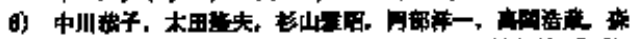

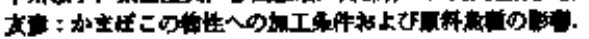
日大당. 14, 501-507 (1997).

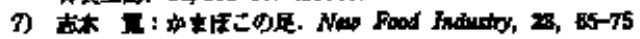
(1981).

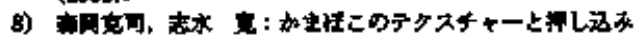

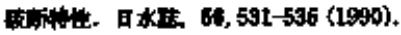

9) H. Topham, K. Serniti, M. Kinonhite, Y. Shimith and M.

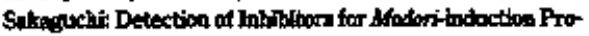

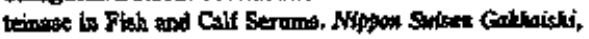
50, 521-5es (1991). 